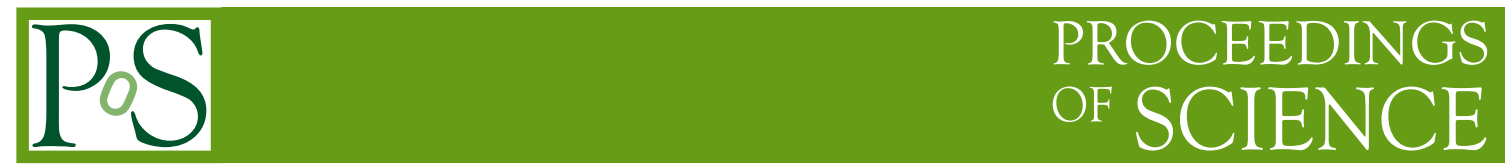

\title{
Advances in Detector Technology
}

\author{
James E. Brau ${ }^{* \dagger}$ \\ University of Oregon, Eugene, USA 97403-1274 \\ E-mail: jimbrau@uoregon.edu
}

This review provides an overview of many recent advances in detector technologies for particle physics experiments. Challenges for new technologies include increasing spatial and temporal sensitivity, speed, and radiation hardness while minimizing power and cost. Applications are directed at several future collider experiments, including the Large Hadron Collider luminosity upgrade (sLHC), the linear collider, and the super high luminosity B factory, as well as neutrino and other fixed target experiments, and direct dark matter searches. Furthermore, particle physics has moved into space, with significant contributions of detector technology, and new challenges for future efforts.

European Physical Society Europhysics Conference on High Energy Physics, EPS-HEP 2009, July 16 - 222009

Krakow, Poland

*Speaker.

${ }^{\dagger}$ Supported by grants from the U.S. Department of Energy and National Science Foundation 


\section{Introduction}

Advances in detector technology enable sharpened understanding and discovery in particle physics. As accelerator technology, and other innovations such as detectors in space, advance the scientific frontier, it remains essential that detector advances keep pace. Detector advances also rejuvenate the scientific programs of established accelerator facilities.

Scientific opportunities abound, but pose great challenges for detectors. The Large Hadron Collider luminosity upgrade (sLHC)[1], the International Linear Collider (ILC)[2], the Super B Factory[3], neutrino experiments[4], direct dark matter searches[5], and astroparticle physics experiments[6] all will be limited by the degree of success in detector R\&D. Many have common challenges and will benefit from parallel advances.

Significant effort is being devoted to developing detector technology. This summary is necessarily incomplete due to time and space limitations, and the author apologies for choices and omissions.

\subsection{Challenges}

The challenges to the experimenter are numerous. Ever increasing precision in energy, momentum, space, and time is needed. Detectors must be fast, with minimal occupancy. Radiation hardness is demanded, as is background rejection. Power and cooling constrain detector design, and must be dealt with. Cost constraints impose practical limitations. These and other considerations motivate efforts to surpass all current performance limits.

Details on many advances and developments in progress are reported in contributions to recent detector conferences, including the Dresden IEEE Nuclear Science Symposium[7], TIPP09[8], and the latest Pisa meeting[9].

\subsection{Enabling Advances}

Improvements in detector technology often come from capitalizing on industrial progress. This happens now at an accelerated pace. Ever finer feature size becomes possible, with detector elements of $10 \mu \mathrm{m}$ or less now feasible. Likewise, faster, low noise, low power electronics is commonly available. Integration advances in microelectronics and mechanics enable novel, complicated instrumentation. New material developments create opportunities for detector designs which are highly radiation resistant, robust, thin, and durable. Understanding damage and annealing mechanisms increases hardness to very high radiation dose levels.

\subsection{The Enterprise}

The particle physics detector community works at several differing scientific fronts, with distinct challenges, requirements, and solutions. For colliders, detector applications address tracking needs for vertex and outer tracking systems, calorimeters, and particle identification (including muon detectors). Quite different challenges appear for direct dark matter experiments. In another thrust, neutrino detectors aim for the largest mass. Finally, the field has moved into particle astrophysics experiments on Earth and in space, creating yet additional issues. These diverse applications require many different approaches. 
Advances build on the core technologies. These include solid state tracking detectors, gaseous detectors, crystals, cryogenic liquids, readout electronics, services (including power, cooling, support, and materials), metrology, and trigger and data acquisition. Advances on all fronts are needed, in parallel, to advance the scientific frontier.

\section{Overview of Experimental Programs}

A diverse portfolio of experimental programs drives detector advances. These include the LHC experiments, the future linear collider, heavy flavor experiments, neutrino experiments, direct dark matter detection experiments, and operations in space.

\subsection{LHC Detectors}

Successful construction and commissioning of the LHC detectors established critical lessons for the future. The detectors are now operating, and planning is underway for upgrades to deal with increasing LHC luminosity. A luminosity of $10^{35} \mathrm{~cm}^{-2} \mathrm{~s}^{-1}$ is anticipated for the sLHC at the end of the decade. Such a luminosity will require innovations, particularly for the inner trackers, where a complete replacement will be required. Radiation damage limits are stretched, and rates exceed the limits for some candidate technologies, requiring increased granularity. In addition to upgrades for the inner tracking, other systems will also need to be improved, especially the electronics.

\subsection{Linear Collider Detectors}

The linear collider detectors will require exceptional precision, with time stamping to suppress backgrounds. The ILC is designed for a bunch train of about about 3000 bunches over 1 msec. The goal for vertex detector precision is $<4 \mu \mathrm{m}$, based on $\sim 20 \mu \mathrm{m}$ pixels in order to optimize heavy flavor tagging. Momentum resolution must be $\sigma(1 / \mathrm{p}) \sim$ few $\times 10^{-5}$ to reconstruct the Higgs as a missing mass in the Higgstrahlung channel. Physics requirements (W/Z separation in the dijet channel) set the jet resolution requirement of $\sigma\left(\mathrm{E}_{j e t}\right) / \mathrm{E}_{j e t} \sim 3-4 \%$ for $\mathrm{E}_{j e t}>100 \mathrm{GeV}$.

\subsection{Heavy Flavor Experiments}

LHCb will soon commence operation. It will be challenged by high radiation levels, a particular problem for the vertex locator (VELO), which is expected to eventually need replacement.

Efforts toward a Super B Factory aim at reduced multiple scattering in the tracker with thinner detectors, rad-hard endcap crystals, and advanced end-cap particle identification techniques.

NA62 aims to measure the rare decay $K^{+} \rightarrow \pi^{+} v \bar{v}$, having developed a state-of-the-art gigatracker and a RICH designed to suppress the $K^{+} \rightarrow \mu^{+} v$ background by three orders of magnitude (after a kinematic suppression of five orders of magnitude and another five orders of magnitude suppression from penetration measurements.)

MEG employs a liquid xenon calorimeter in the search for $\mu \rightarrow \mathrm{e} \gamma$. It demands high purity and good understanding of the response and calibration. 


\subsection{Neutrinos}

Neutrino experiments are benefiting from recent technological advances. The development of multi-pixel photon counters (MPPCs, or silicon photomultipliers - SiPMs) is an important one. T2K employs about 60,000 devices to read out the scintillator bars in the near detector. NOvA is constructing a massive (16 kton) extruded CVC contained liquid scintillator detector in search of $v_{\mu} \rightarrow v_{e}$.

Looking toward the future, megaton-scale detectors are envisioned. For the full scale water Cherenkov detector, innovations in photomultiplier tubes to manage costs are needed. A somewhat less massive detector with tracking liquid argon may achieve the physics goals.

\subsection{Direct Dark Matter Detection}

Direct dark matter detection experiments search for very weakly interacting particles, which requires extremely sensitive detector elements, with a threshold of just a few keV. Background suppression is essential, driving the experiments deep underground, with passive shields, and detectors built with low intrinsic radioactivity, and capable of gamma background discriminatation. Experimenters look for signatures based on ionization, scintillation, phonons, or a combination.

\section{Technologies}

Advances in detectors span a wide spectrum of technologies. There are notable recent advances in silicon and diamond tracking detectors, gaseous tracking detectors, electromagnetic and hadronic calorimeters, detectors for particle identification, progress toward megaton neutrino detectors, and dark matter detectors.

\subsection{Solid State Tracking Detectors}

The construction and commissioning experience of the LHC detectors[10, 11] and the Fermi Gamma-ray Space Telescope[12](Figure 1) provided many lessons for large scale silicon systems. The next challenges will include the increased rate and radiation levels of the sLHC, the increase precision demanded by the ILC and the B Factories, and a variety of specialized applications, such as the NA62 Gigatracker.

The event rate at the sLHC will be about 300-400 events per crossing, with about 10,000 tracks within $|\eta| \leq 3.2$. The intense radiation levels[13] posed by the sLHC for tracking are $10^{16} / \mathrm{cm}^{2}$ $(\sim 400 \mathrm{MRad})$ at a radius of $5 \mathrm{~cm}$ from the beamline, $10^{15} / \mathrm{cm}^{2}(\sim 40 \mathrm{MRad})$ at a radius of 20 $\mathrm{cm}$, and $2 \times 10^{14} / \mathrm{cm}^{2}(\sim 10 \mathrm{MR})$ at a radius of $50 \mathrm{~cm}$ (See Figure 2$)$. These levels dictate the technologies under consideration[13]. Beyond $60 \mathrm{~cm}$ conventional silicon strips are suitable, but within $60 \mathrm{~cm}$ short strip detectors must be used. Within $20 \mathrm{~cm}$ pixel technologies are required, while within $\sim 10 \mathrm{~cm}$, even the standard pixel technology is unacceptable, and new technologies are being developed. ATLAS considers several candidate technologies for this innermost region, including planar technology, 3D-silicon, diamond[14], and GOSSIP[15], a gaseous pixel detector (see Figure 3). Radiation damage studies of diamond are presented in Figure 4.

Development of silicon sensors for linear collider vertex detectors confront a different set of requirements. Radiation levels will be much lower, but the physics and machine demands call for 


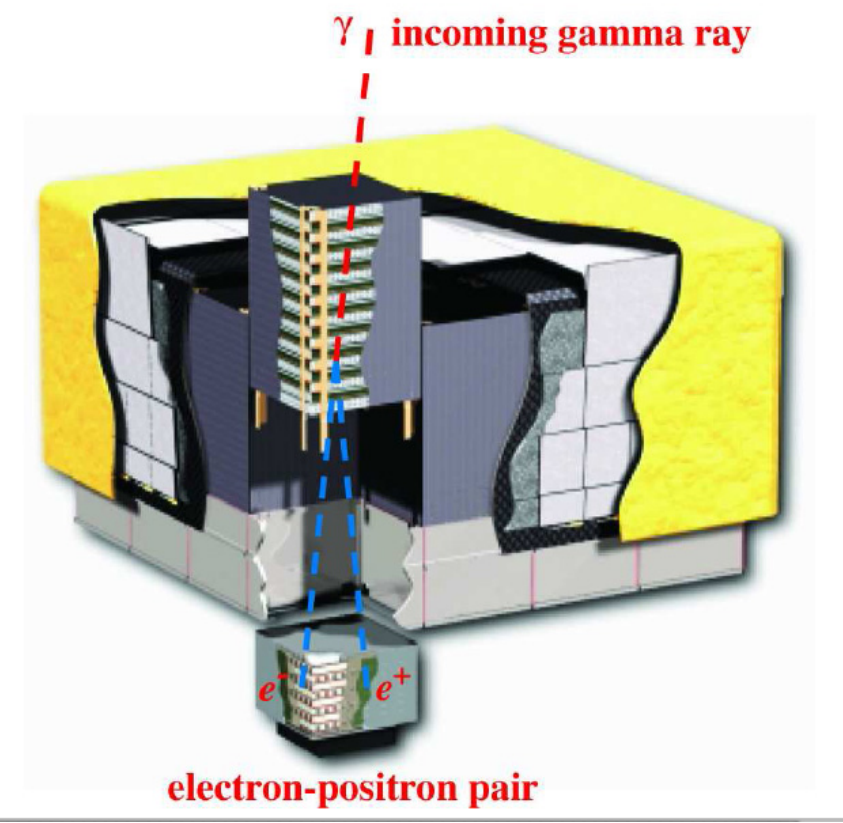

Figure 1: Schematic diagram of the Fermi Large Area Telescope[12]. The converter-tracker has 16 planes of high-Z material interleaved with tracking planes of single-sided silicon strip detectors. The overall dimensions of the telescope are $1.8 \mathrm{~m} \times 1.8 \mathrm{~m} \times 0.72 \mathrm{~m}$.

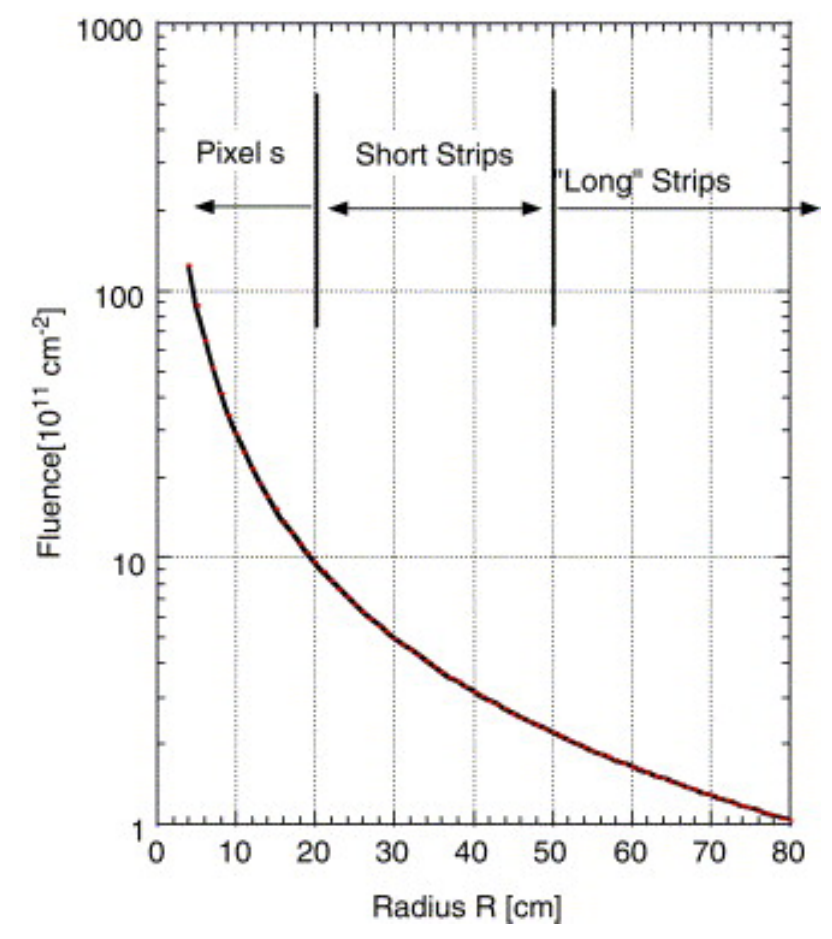

Figure 2: Expected radial fluence as a function of radius for an SLHC detector after an integrated luminosity of $2500 \mathrm{fb}^{1}$, with radial extent of proposed tracker regions. [13] 


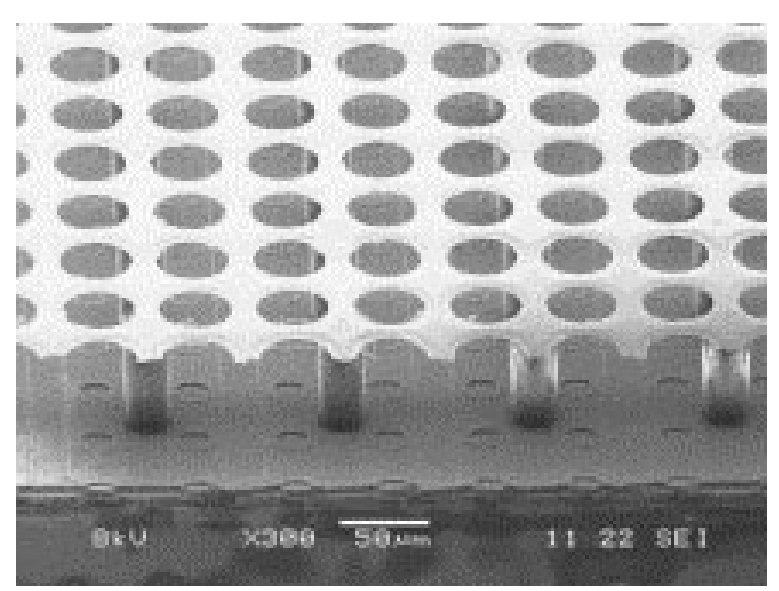

Figure 3: The Integrated Grid (InGrid) on top of a 20 $\mu \mathrm{m}$ SiProt layer, on top of a TimePix chip[15].

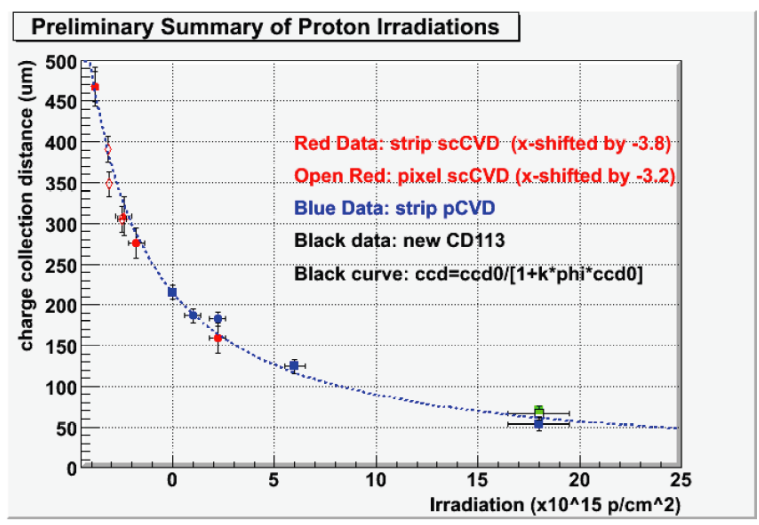

Figure 4: Proton irradiation results for $\mathrm{pCVD}$ (blue points) and scCVD (red points) material at $1 \mathrm{~V} / \mathrm{m}$ and $2 \mathrm{~V} / \mathrm{m}$ (green square). See reference [14] for interpretation.

- excellent spacepoint precision ( $<4$ microns),

- superb impact parameter resolution $\left(5 \mu \mathrm{m} \oplus 10 \mu \mathrm{m} /\left(\mathrm{p} \sin ^{3 / 2} \theta\right)\right)$,

- transparency $\left(\sim 0.1 \% \mathrm{X}_{0}\right.$ per layer $)$,

- track reconstruction (find tracks in the vertex detector alone),

- sensitivity to minimal bunch crossings ( $<150$ crossings $=45 \mu \mathrm{sec}$ for ILC),

- EMI immunity, and

- limited power $(<100$ Watts for passive cooling to minimize material).

Several efforts are addressing these challenging requirements. Concepts under development include charge-coupled devices (CCDs), which were successfully applied in the 307 Megapixel SLD vertex detector[16], as well as several other approaches. CCDs as implemented by SLD are too slow for the ILC. The efforts to advance the technology include[17]:

- column parallel readout CCDs,

- FPCCDs (fine pixel CCDs),

- monolithic active pixels based on CMOS (MIMOSA, MAPs, FAPs, Chronopixels, 3D-SOI),

- DEPFET (DEpleted P-channel Field Effect Transistor ),

- SoI (silicon on insulator),

- ISIS (Image Sensor with In-Situ Storage), and

- HAPS (hybrid active pixel sensors). 
Silicon tracking is also under development for the linear collider by the SiD design group[18]. The superb resolution of silicon allows a small tracking volume to achieve the tracking goals of the ILC, better than $\sigma_{p} / \mathrm{p}=1 \%$ at $100 \mathrm{GeV} / \mathrm{c}$. Silicon is fast, and provides robustness to backgrounds and occasional beam disruptions anticipated at the linear collider. The performance goal requires very low mass support so as to minimize the material within the tracking volume. The $\mathrm{SiD}$ design of modular low mass sensor tiles of carbon fiber cylinders achieves $0.6 \%$ of a radiation length per layer, with passive cooling. The anticipated material budget of the inner tracking system (vertex and tracker) is less than $10 \%$ of a radiation length for $\theta>50^{\circ}$.

A silicon envelope for a TPC tracker is being studied by the SiLC Collaboration[19]. This system is envisioned to provide improved tracking performance, tracking hermiticity, monitoring and alignment, and robustness.

The Gigatracker[20] under development for NA62 employs three silicon pixel sensors to provide precise direction and timing information. A gigaHertz rate with more than a megaHertz/mm 2 at the maximum must be handled in vacuum. Two readout options are being developed, a constant fraction discriminator (CFD) with complex pixel circuitry, and a time over threshold (TOT) with simple, low power pixel circuitry. Acceptable prototypes of the analog circuits for both options have been produced in $0.13 \mu \mathrm{m}$ CMOS.

Diamond has certain properties that give it a potential advantages over silicon as a sensor material. These include a larger bandgap and the consequent low leakage current, the strong atomic bonds which make for high radiation hardness, and the smaller dielectric constant. Chemical vapor deposition diamond is available as single-crystal (scCVD) or polycrystalline (pCVD) diamond. pCVD wafers can be grown to $12 \mathrm{~cm}$ diameter and $2 \mathrm{~cm}$ thick. scCVD diamonds with an area of a few $\mathrm{cm}^{2}$ and about $1 \mathrm{~mm}$ thickness are available. Over the past several years, experience with diamond as radiation monitors have been achieved, and it now appears as a candidate for the LHC inner tracking (mentioned above)[14].

\subsection{Gaseous Tracking Detectors}

The ALICE time projection chamber (TPC) is the largest TPC ever built. It has an outer radius of $2466 \mathrm{~mm}$, and a drift length of 2 x $2500 \mathrm{~mm}$ drift[21].

Recent work has been aimed at developing micropattern gas detectors (MPGDs)[22] as replacements for the conventional gaseous TPC readout. MPGDs are immune to $\mathrm{E} \times \mathrm{B}$ effects that plague conventional detectors. This work includes GEMs, MicroMeGas, and the Timepix (CMOS)/Ingrid approach. To date, the T2K off-axis near detector is the largest TPC equipped with MPGDs. The three T2K TPCs (Figure 5) consist of 72 Micromegas modules, 12 modules per readout plane, with $9 \mathrm{~m}^{2}$ active area comprising 120k electronics channels[26]. The magnetic field is 0.2 Tesla.

The LCTPC Collaboration is studying a large TPC (720 mm inner diameter field cage) with MPGDs in beam[23] as a prototype for an ILC detector[24]. Both double GEMS and Micromegas have been tested within a 1 Tesla field. A triple GEM structure with Timepix readout has also been tested[25].

\subsection{Electromagnetic Calorimetry}

Notable recent work on electromagnetic calorimetry has included silicon-tungsten, scintillator 


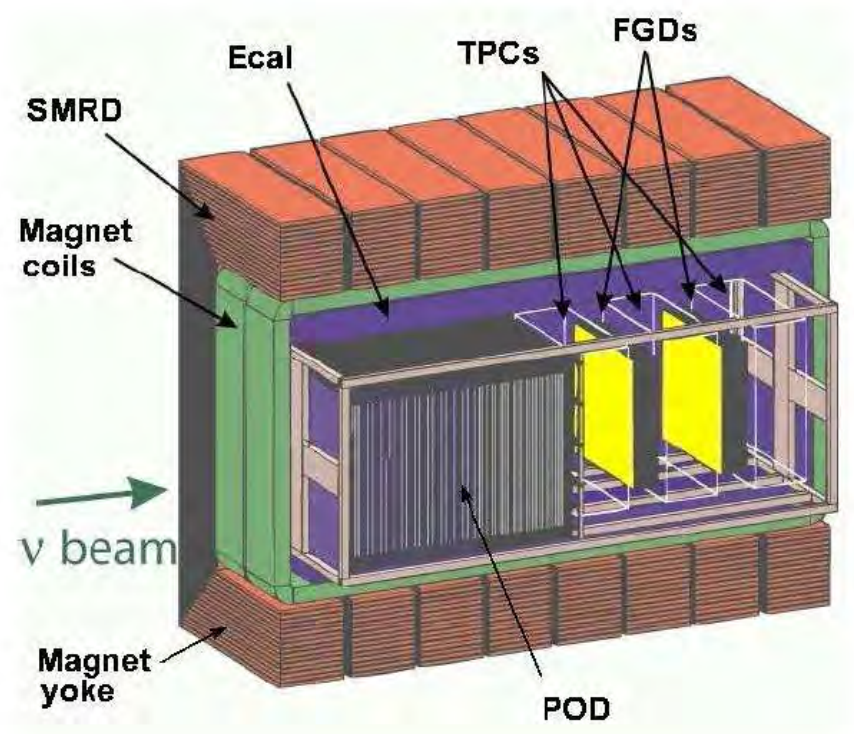

Figure 5: Cutaway view of the T2K off-axis near detector[26].

strips, crystals, and liquid xenon for MEG.

A fine grained electromagnetic calorimeter serves several goals toward optimized physics performance of the ILC experiments. It is critical to the success of the particle flow technique for hadron jet calorimetry, but also important for other physics, such as the tau decay reconstruction. A natural technology choice is the silicon-tungsten ( $\mathrm{SiW}$ ) sampling calorimeter. Good success was achieved using SiW for luminosity monitors at SLD, DELPHI, OPAL, and ALEPH [27, 28, 29, 30, 31]. SiW sampling calorimetry specifically optimized for the ILC experiments is under development by two groups, the SiD design group, and the CALICE Collaboration.

CALICE has been conducting a series of beam tests over the past few years[32]. Beginning at DESY in 2006, the tests continued at CERN (2006-7), and at Fermilab in 2008-9. The data from 6 $\mathrm{GeV}$ to $25 \mathrm{Gev}$ shows a resolution of

$$
\frac{\sigma_{E}}{E}=\frac{(16.53 \pm 0.14(\text { stat }) \pm 0.4(\text { syst })) \%}{\sqrt{E(G e V)}} \oplus(1.07 \pm 0.14(\text { stat }) \pm 0.1(\text { syst })) \%
$$

The collaboration is also building a technological prototype, which will integrate the mechanical, thermal, and electric constraints for an ILD electromagnetic calorimeter module, include embedded readout electronics, and allow for a realistic study of the construction processes.

The SiD team (a SLAC/Oregon/BNL/Davis/Annecy Collaboration) is developing a very dense, fine grained silicon tungsten calorimeter for the ILC, with a pad size of $13 \mathrm{~mm}^{2}$ to match the Moliere radius ( $\left.\sim \mathrm{R}_{M} / 4\right)$ [33]. Each six inch wafer of 1024 pads is read out by one integrated chip (KPiX). Tests have demonstrated that less than $1 \%$ crosstalk is expected. A KPiX design[34] has been fabricated and is under test. In order to be sensitive to single minimum ionizing particles, the signal-to-noise must exceed seven, making the required noise level less than 2000 electrons. A dynamically switchable feedback capacitor scheme achieves the required dynamic range of 0.1- 
2500 MIPs. The mechanical design calls for passive cooling, with heat conducted through the tungsten to the edge of each module. The collaboration is working toward a prototype tower of the pad structure. A MAPS version is also under development in collaboration with RAL[35].

The CALICE Collaboration has tested a scintillator strip electromagnetic calorimeter read out by multi-pixel photon counters in a beam at DESY and Fermilab. It is designed for the particle flow technique at the ILC, where 3 - $5 \mathrm{~mm}$ strips for high granularity are needed.

Crystals have a long history in electromagnetic calorimetry[36], and new HEP calorimeter applications are now under development. Applications include PWO for PANDA at GSI, LYSO (Cerium doped Lutetium Yttrium Orthosilicate, $\mathrm{Lu}_{2(1-x)} \mathrm{Y}_{2 x} \mathrm{SiO}_{5}$ :Ce) for a Super B Factory, Mu2e and the CMS endcap upgrade, and $\mathrm{PbF}_{2}, \mathrm{BGO}$, or $\mathrm{PWO}$ for a homogeneous hadron calorimeter. The SuperB/Mu2e/CMS endcap application demands the highest possible radiation hardness. LYSO is currently favored for its radiation hardness, large light yield, and low noise. The homogeneous hadron calorimeter (HHCAL) application is an interesting recently advanced crystal concept[37] describe below.

The MEG experiment at the PSI employs an 800 liter liquid xenon calorimeter, with 846 PMTs. It now starts a new run with improved performance. Superb resolutions in energy, position, and time are possible[38].

\subsection{Hadronic Calorimetry}

Recent interest in particle flow calorimetry for the ILC drives advances on several technologies. The fundamental idea of particle flow calorimetry is to capitalize on the excellent tracker momentum measurement of the charged component of a jet, and to measure only neutral components of the jet in the calorimeter. These separately measured components can be combined for an optimal jet energy measurement. This technique requires excellent separation of the calorimeter depositions from neutral and charged particles with a highly granular calorimeter. The hadron calorimeter detector technologies of interest in particle flow calorimetry include analog scintillator calorimeters with silicon photomultiplier (SiPM) readout, and digital gas calorimeters, based on RPCs, GEMs, or MicroMeGas[18, 24]. The CALICE Collaboration has constructed and tested a scintillator calorimeter consisting of 38 steel layers ( $2 \mathrm{~cm}$ thick) of about $1 \mathrm{~m}^{2}$ for 4.5 interaction lengths, with 7608 scintillator tiles read out with SiPMs. Each gap in the steel is instrumented with the highly segmented scintillator; the first 30 layers are assembled from tiles varying in area from $3 \times 3 \mathrm{~cm}^{2}$ at the center to $12 \times 12 \mathrm{~cm}^{2}$ (Figure 6). The calorimeter has been tested in beams at DESY, CERN, and Fermilab[39]. The tests included running with a silicon-tungsten electromagnetic calorimeter, and a tail catcher, muon tagger (TCMT) backup, and featured common readout electronics. The CALICE Collaboration draws the following conclusions from these tests: 1.) the SiPM technology has proven to be robust and stable, 2.) the calibration is well under control, 3.) the performance is as expected and understood, and 4.) the results strongly support the predicted particle flow performance.

A small glass RPC calorimeter has been tested at Fermilab[40], and a large calorimeter designed for particle flow is under construction. The tested RPC module was built of $20 \mathrm{x} 20 \mathrm{~cm}^{2}$ RPCs with $1 \times 1 \mathrm{~cm}^{2}$ readout pads. The 10 chambers consisted of 2560 readout channels. The beam tests involved $120 \mathrm{GeV}$ protons, and $1-16 \mathrm{GeV} \pi^{+}$and $\mathrm{e}^{+}$. A one $\mathrm{m}^{3}$ prototype is under construction, with beam tests and analysis planned in 2010-11. 


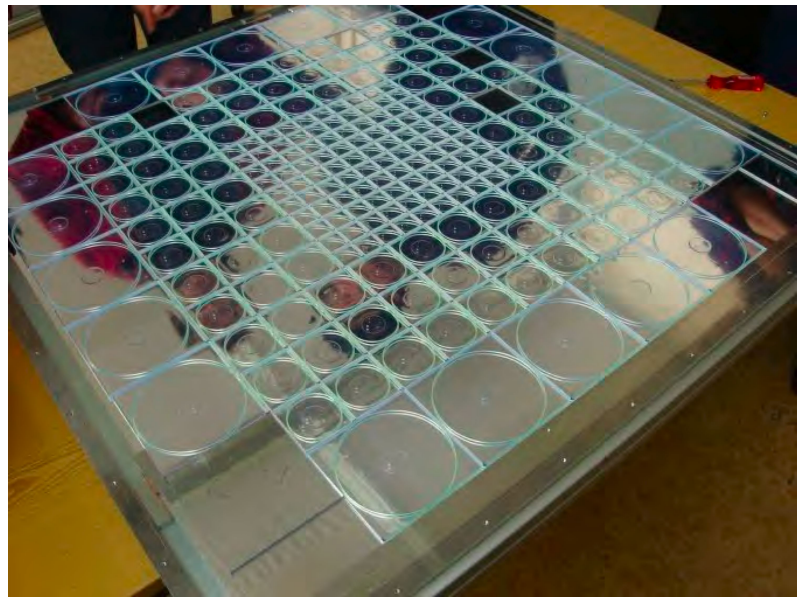

Figure 6: CALICE scintillator hadron calorimeter layer. [39]. The pattern is built from 216 tiles.

Since the fluctuations in hadronic showers are dominated by nuclear binding energy losses and $\pi^{0}$ energy fluctuations, the resolution of a hadron shower can be improved by separately measuring the energy deposited by electromagnetic showers resulting from the $\pi^{0}$ s from the energy of non-relativistic particles[41]. The DREAM Collaboration has realized a measurement of this with a copper fiber calorimeter, where quartz and scintillating fibers separately measure these two components[42]. While the constructed calorimeter is leakage limited for high energy showers, the collaboration is able to demonstrate improved resolution when the two components are combined. Figure 7 shows the response in the DREAM calorimeter to $100 \mathrm{GeV} \pi^{-}$. The Cherenkov and scintillator signals are shown to respond differently to the electromagnetic content of the showers.

The homogeneous hadron calorimeter (HHCAL)[37] would operate in the dual readout mode, separating the signals from relativistic particles and non-relativistic particles by detecting Cherenkov and scintillation light separately. Large volume is required, so cost effectiveness is essential. The crystal material must be UV transparent. Three candidates have been evaluated $\left(\mathrm{PbF}_{2}, \mathrm{BGO}\right.$, $\mathrm{PWO}$ ), and initial investigation favors scintillating $\mathrm{PbF}_{2}[43]$.

The sLHC's harsh radiation environment will pose an increased challenge for the forward calorimeters. For example, the liquid argon in the ATLAS forward calorimeter will heat and may boil, and space charge will build up between electrodes. These effects could require new forward calorimeters. ATLAS is confronting this challenge with the development of two possible solutions. A new forward calorimeter, with smaller gaps and increased cooling would replace the existing calorimeter, to handle the radiation. Such a calorimeter is under development[44]. This solution requires opening the cryostat. An alternative solution would be to insert a warm calorimeter in front of the current calorimeter.

\subsection{Particle Identification}

Particle identification has been an important element in recent experiments (BaBar, Belle, LHC-b), and continues to be a focus in detector development for future heavy flavor experiments (Belle II[45], INFN Super B, NA62). The key technologies are radiators and photodetectors. 


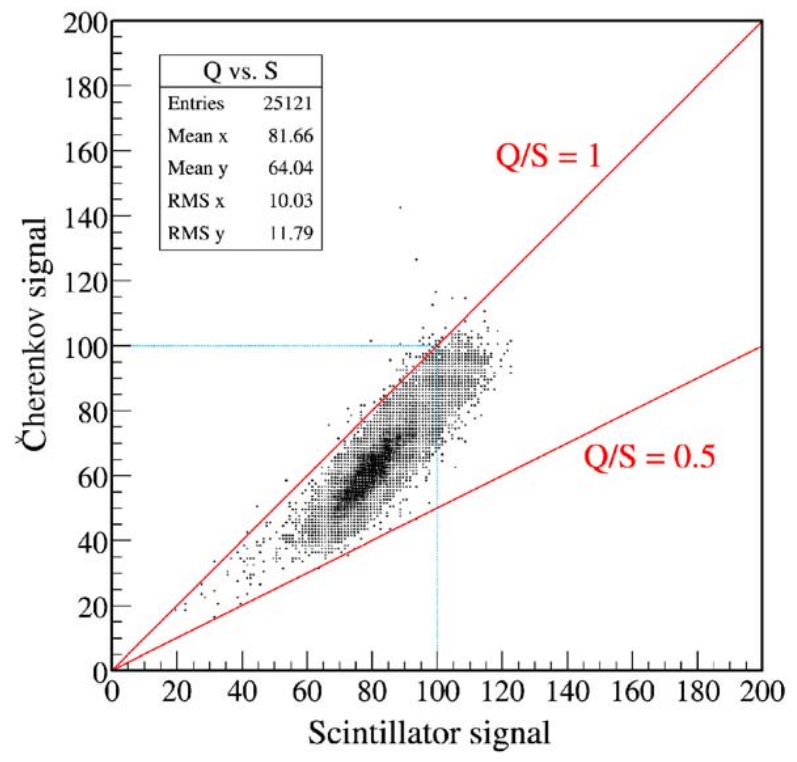

Figure 7: Cherenkov signals versus scintillator signals for $100 \mathrm{GeV} \pi^{-}$in the DREAM Calorimeter [42].

Polishing techniques are advancing for quartz radiators, and silica aerogel developments include multi-index tiling. The photodector technology is advancing, including the hybrid photodectors, microchannel plates with phototubes, and multi-pixel photon counters (MPPCs or SiPMs). The MPPCs are single photon sensitive devices, built from avalanche photodiodes (APD) arrays on a common silicon substrate. They are extremely compact, B field immune, provide very good timing, and offer gain and quantum efficiency competitive with a PMT. There are many developments and investigations pushing this technology. Notable are the MPPCs for the fiber readout of the T2K near detectors, fabricated by Hamamatsu, which contain 667 pixels $\left(50 \times 50 \mu \mathrm{m}^{2}\right)$ on $1.3 \mathrm{~mm} \times$ $1.3 \mathrm{~mm}$ arrays. More than 60,000 arrays will be used to read out all detectors except the TPCs[46].

\subsection{Megaton Detectors for Neutrinos}

The strength of the water Cherenkov technique is well established by the success of Super Kamiokande, and its predecessors. The future goal, aimed at super sensitive neutrino mixing and proton decay experiments, is the megaton detector. The challenges are costs, improved quantum efficiency for the PMTs, readout electronics, new photosensors, and hardening against unexpected accidents.

A promising large new photo-detector under development[47] is the Hybrid Avalanche Photodetector (HAPD), offering potentially easier construction due to its simpler structure, and therefore simplified mass production and lower manufacturing cost. A 13-inch version has been successfully developed and tested[48]. The internal surface of the HAPD glass envelope is coated with a photocathode and a light reflector. The very high voltage $(\sim+20 \mathrm{kV})$ used for acceleration of electrons toward an avalanche diode results in a high gain $\left(\sim 10^{5}\right)$. The time resolution is better than that 
of a dynode structure, being about $190 \mathrm{psec}$, compared to the $1.4 \mathrm{nsec}$ of the 13 -inch PMTs. The elimination of a costly dynode structure, in favor of an avalanche diode, reduces cost (Figure 8).

A parallel path toward the megaton-class neutrino detector is offered by the liquid argon TPC. ICARUS has demonstrated the potential of this approach, with 300 kilogram chambers (Figure 9)[49]. With its low threshold, a 100 kton device is competitive with the megaton water Cherenkov. The challenges include purification, low noise electronics operating cold with signal multiplexing, design of the vessel including materials and insulation, underground siting, and costs[4].

\subsection{Neutrinoless Double Beta Decay}

Neutrinoless double beta decay holds the potential to establish the mass scale of the neutrinos. Several 100-200 kg detectors are now being developed. The major challenge is to minimize backgrounds. Among the promising efforts are CUORE[50], EXO[51], and Majorana[52]. CUORE is a $203 \mathrm{~kg}{ }^{130} \mathrm{Te}$ system composed of $988 \mathrm{TeO}_{2}$ bolometers. This effort builds on the $11 \mathrm{~kg}{ }_{130} \mathrm{Te}$ CUORICINO effort[53]. EXO is developing a $200 \mathrm{~kg}{ }^{136} \mathrm{Xe}$ chamber, in which ionization and scintillation are measured, and Ba tagging will be added (EXO-200). Majorana is working toward

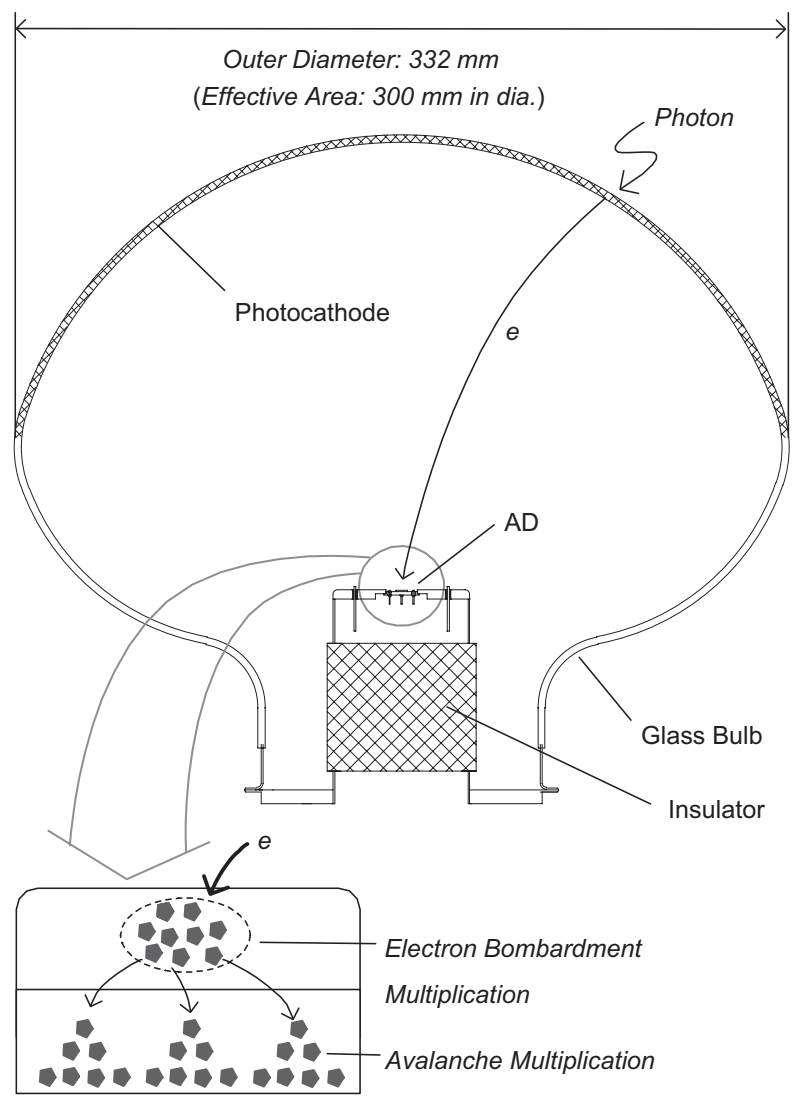

Figure 8: Outline of the large-aperture Hybrid Avalanche Photo-detector (HAPD) and its principle of operation[47]. 


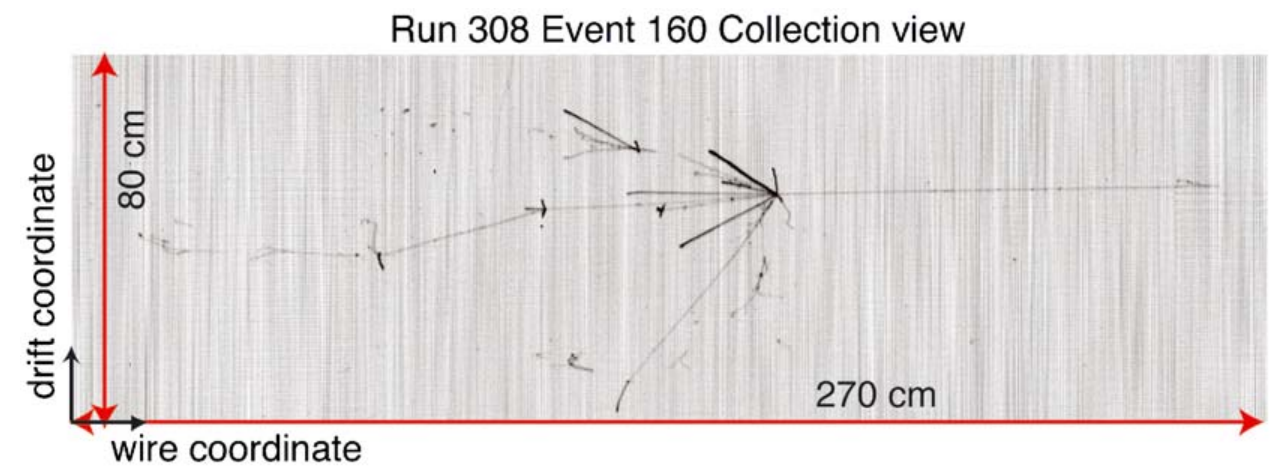

Figure 9: A hadron shower in the ICARUS detector [49]

a $120 \mathrm{~kg}{ }_{76} \mathrm{Ge}$ experiment. These, and other efforts, are leading the way toward few milli-eV $v_{e}$ sensitivity.

\subsection{Dark Matter Direct Detection Techniques}

Given the very small interaction rate expected of dark matter particles (WIMPs), detector advances strive for large masses and low thresholds ( few $\mathrm{keV})$. The experimental strategy can employ signals from ionization, scintillation, phonons, or a combination.

Several bolometers operate at cryogenic temperatures with state of the art techniques. CDMS (Cryogenic Dark Matter Search) uses germanium and silicon ZIP detectors[54] to collect phonons and charge(Figure 10). With highly segmented readout elements, and good relative timing of ionization and phonon signals, good event localization is possible. A five tower, $\sim 5.5 \mathrm{~kg}$ cryogenic assembly has operated in the Soudan mine, and a $25 \mathrm{~kg}$ SuperCDMS is being planned for SNOLAB[56].

Examples of other active bolometers include EDELWEISS, CRESST-II, and ROSEBUD. EDELWEISS has searched for interactions in germanium with $\mathrm{Ge} / \mathrm{NTD}, \mathrm{Ge} / \mathrm{NbSi}$, and $\mathrm{Ge} / \mathrm{Interdigit}$ detectors. EDELWIEISS now operates thirty $\mathrm{kg}$ under the Frejus mountain, in the underground laboratory of Modane at the French-Italian border. CRESST-II watches 300 grams of $\mathrm{CaWO}_{4}$ crystal in Gran Sasso, monitoring scintillation and phonons. ROSEBUD is developing a cryogenic, crystal scintillator of BGO.

The warm liquid bubble chamber [57] of COUPP (Chicagoland Observatory for Underground Particle Physics) offers a different approach in the search for dark matter. Two kilograms of $\mathrm{CF}_{3} \mathrm{I}$ has operated successfully (Figure 11). New 20 and $60 \mathrm{~kg}$ chambers are being constructed, and will go underground in 2010.

Another strategy to background reduction is the detector that uses the recoiling atoms to determine the dark matter particle direction of origin, providing a suppression of the isotropic background[58]. Low pressure TPCs are favored for such "directional dark matter detectors." $\mathrm{CS}_{2}$ is sensitive to spin-dependent interactions, and $\mathrm{CF}_{4}$ and ${ }^{3} \mathrm{He}$ are sensitive to spin-independent interactions. Different readout approaches are in use. DRIFT-II uses wire chambers, with two $1 \mathrm{~m}^{3}$ $\left(\mathrm{CS}_{2}\right)$ modules now underground. NEWAGE and MIMAC are employing MPGDs, while DMTPC uses PMT and CCD readout with a $\mathrm{CF}_{4}$ detector (Figure 12). 


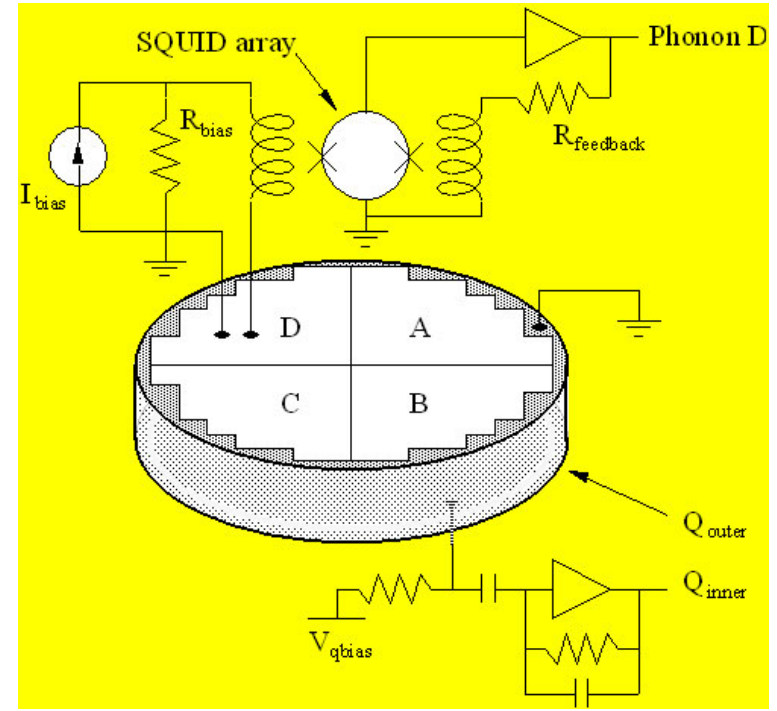

Figure 10: Schematic of the ZIP detector of CDMS, illustrating the four phonon and two ionization channels along with their readout electronics[55].

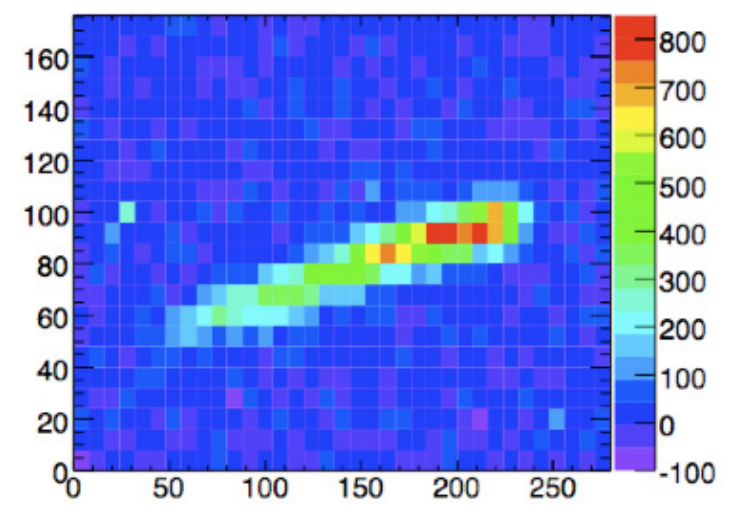

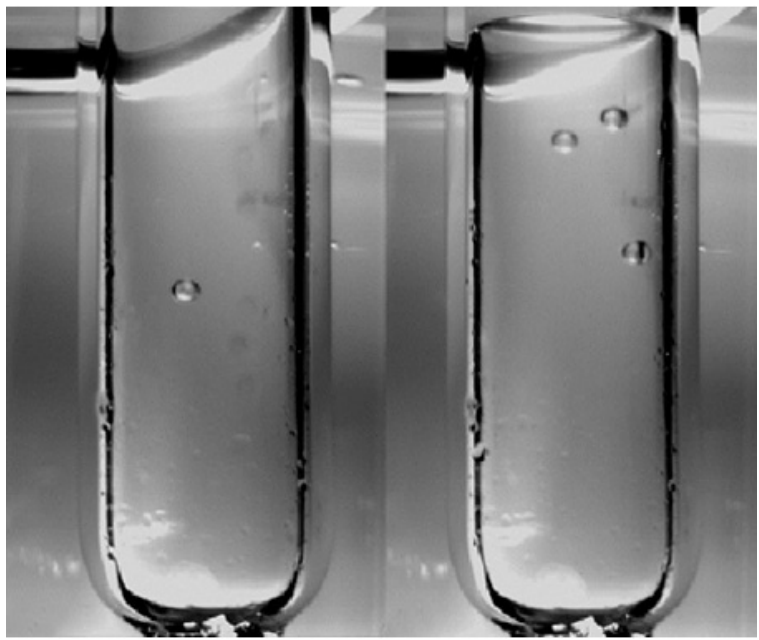

Figure 11: Images from the COUPP bubble chamber. A single bubble like that expected from WIMPs (left), and several simultaneous disconnected bubbles (right), a signature of neutron recoils. [57].

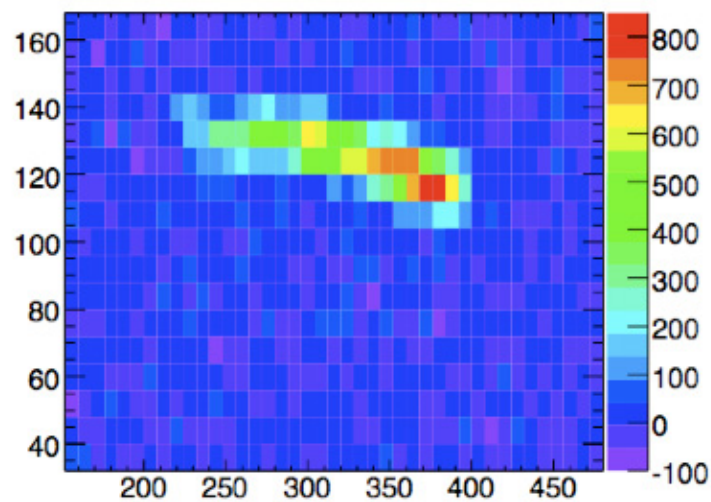

Figure 12: Nuclear recoil candidates of DMTPC[59] induced by neutrons incident from the right. 100 pixels correspond to $6 \mathrm{~mm}$.

Nobel liquids (argon, xenon[60], neon) bring many attractive features to the pursuit of dark matter detection: relatively low cost, ease to obtain, high density as target material, easily purified due to freeze out of contaminants at cryogenic temperatures, very small electron attachment probability, large electron mobility (that is, large drift velocity for small E-field), high scintillation efficiency, and possibility for large, homogenous detectors. The fundamental limitation and problem is the presence of radioactive ${ }^{39} \mathrm{Ar}$ and ${ }^{85} \mathrm{Kr}$. Efforts include both single phase techniques, and the two phase approach. DEAP/CLEAN[61] and XMASS[62] are examples of the single phase work, and XENON[63], LUX[64], and ArDM[65] are examples of the two phase approach. 


\subsection{Test Beams}

Test beams are an essential tool for the development of advanced detectors. Such beams are important to many other phases of the particle physics experiment as well, such as prototype testing, calibrations, etc. But their value to the early phase of detector development is essential, and laboratory support for this is critical, and appreciated by the community.

\section{Conclusions}

Discoveries in particle physics vitally depend on advances in detector technology. The challenges confronting the experimenter today are huge: greater speed, finer granularity, higher radiation hardness, more exotic materials, etc. Many efforts are addressing these challenges. It is critical to the future of the field that these efforts be well funded. With support, the technology will advance, providing emerging capabilities critical to future discoveries.

\section{Acknowledgments}

The author is pleased to acknowledge assistance of colleagues in the preparation of this presentation, including E. Aprile, M. Breidenbach, A. Bevan, K. Dehmelt, M. Demarteau, B. Fleming, G. Gratta, D. Hitlin, J. Jaros, H. Ma, G. Rakness, J. Repond, F. Sefkow, A. Seiden, D. Strom, F. Taylor, J. Timmermans, D. Wark, and A. White.

This work was sponsored in part by the US Department of Energy and the US National Science Foundation.

\section{References}

[1] M. Mangano, The super-LHC, [http://arXiv.org/pdf/0910.0030].

[2] J. Brau (ed. )et al., International Linear Collider reference design report. 1:Executive summary. 2: Physics at the ILC. 3: Accelerator. 4: Detectors, ILC-REPORT-2007-001, [http://arXiv.org/pdf/0712.1950].

[3] Y. Sakai, Super B Factories, PoS FPCP2009 (2009) 022.

[4] B. Fleming, Experimental Overview of Neutrino Properties, DPF Meeting, 2009.

[5] E. Aprile and S. Profumo, Focus on dark matter and particle physics New J. Phys. 11 (2009) 105002.

[6] A. Olinto, Particle Astrophysics, High Energy Gamma-ray and Neutrino Astronomy, DPF Meeting, 2009.

[7] IEEE Nuclear Science Symposium, Dresden, October, 2008.

[8] TIPP09, Tsukuba, March, 2009.

[9] 11th Pisa Meeting, May, 2009.

[10] The ATLAS Collaboration, G. Aad et al., The ATLAS Experiment at the CERN Large Hadron Collider, JINST 3 (2008) S08003.

[11] The CMS Collaboration, S. Chatrchyan et al., The CMS Experiment at the CERN Large Hadron Collider, JINST 3 (2008) S08004. 
[12] W.B. Atwood et al., The Large Area Telescope on the Fermi Gamma-Ray Space Telescope Mission, The Astrophysical Journal 697 (2009) 1071.

[13] H.F.-W. Sadrozinski and A. Seiden, Tracking detectors for the sLHC, the LHC upgrade, Nucl. Instrum. Meth. A541 (2005) 434-440.

[14] M. Cristinziani, Diamond Prototypes for the ATLAS SLHC Pixel Detector [http://arXiv.org/pdf/0910.0347].

[15] H. van der Graaf et al., New developments in gaseous tracking and imaging detectors, Nucl. Instrum. Meth. A607 (2009) 78-80.

[16] K. Abe et al., Design and performance of the SLD vertex detector: a 307 Mpixel tracking system, Nucl. Instrum. Meth. A400, (1997) 287-343.

[17] C. Damerell et al., ILC Vertex Detector R\&D (2008) ILC-REPORT-2008-016.

[18] H. Aihara (ed.) et al., SiD Letter of Intent [http: / / arXiv . org/pdf / 0911.0006 ].

[19] A. Savoy-Navarro et al., Overview of the SiLC RD activities [http://arxiv.org/pdf/0903.4361].

[20] G. Mazza, The NA62 Gigatracker pixel detector system, Nucl. Instrum. Meth., to be published.

[21] A. Matyja, ALICE TPC-design and performance, Europhysics Conference on HEP, Krakow, 2009.

[22] F. Sauli and A. Sharma, Archana Micropattern gaseous detectors, Ann. Rev. Nucl. Part. Sci. 49 (1999) 341-388.

[23] K. Dehmelt, A Large TPC Prototype for an ILC Detector, Europhysics Conference on HEP, Krakow, 2009.

[24] H. Stoeck et al., ILD Letter of Intent [http://www.ilcild.org/documents/ild-letter-of-intent].

[25] A. Bamberger et al., Resolution studies on 5 GeV electron tracks observed with triple-GEM and MediPix2/TimePix-readout, Nucl. Inst. Meth. A581 (2007) 274-278.

[26] C. Giganti, The T2K TPCs, Europhysics Conference on HEP, Krakow, 2009; also Y. Kudenko, The near neutrino detector for the T2K experiment [http: / / arXiv . org/pdf / 0805.0411 ].

[27] S.C. Berridge et al., First results from the SLD silicon calorimeters, IEEE Trans. Nucl. Sci. 39 (1992) 1242-1248.

[28] D. Strom, Silicon luminosity monitors, in proceedings of the Third International Conference on Calorimetry in High Energy Physics, World Scientific (1993) 357.

[29] D. Bederede et al., SICAL: A High precision silicon - tungsten luminosity calorimeter for ALEPH Nucl. Instrum. Meth. A365 (1995) 117-134.

[30] G. Abbiendi et al., Precision luminosity for $Z^{0}$ lineshape measurements with a silicon-tungsten calorimeter, Eur. Phys. J. C14 (2000) 373-425. [hep-ex/9910066].

[31] S. Almehed et al., A Silicon - tungsten electromagnetic calorimeter for LEP, Nucl. Instrum. Meth. A305 (1991) 320-330.

[32] C. Carloganu, Electromagnetic Calorimetry for the ILC, Europhysics Conference on HEP, Krakow, 2009.

[33] J.E. Brau et al., An electromagnetic calorimeter for the silicon detector concept, Pramana 69 (2007) 1025-1030. 
[34] D. Freytag, KPiX, An Array of Self Triggered Charge Sensitive Cells Generating Digital Time and Amplitude Information, SLAC-PUB-13462 (2008).

[35] J.A. Ballin et al., A digital ECAL based on MAPS [arXiv: 0901.4457$].$

[36] R. Zhu, Precision crystal calorimeters in high-energy physics: past, present, and future, Proc. SPIE 7079 (2008) 70790W.

[37] A. Para, High Resolution Jet Calorimetry: Total Absorption Homogeneous Calorimeter with Dual Readout, Tohoku Linear Collider Workshop, 2009.

[38] Giovanni.Allucci@pi.infn.it, CALOR 2008.

[39] J. Cvach, The CALICE hadron calorimeters - beam test results and new developments, Europhysics Conference on HEP, Krakow, 2009.

[40] B. Bilki et al., Measurement of the Rate Capability of Resistive Plate Chambers, JINST 4 (2009) P06003 [arXiv:0901.4371].

[41] P. Mockett, AReview of the Physics and Technology of High-energy Calorimeter Devices, in proceedings of Dynamics and Spectroscopy At High Energy, Stanford (1983) 335-393.

[42] N.Akcurin et al.,Hadron and jet detection with a dual-readout calorimeter, Nucl. Instrum. Meth. A537(2005) 537-561.

[43] R. Zhu, Crystals for Homogeneous Hadron Calorimetry, the Linear Collider Workshop (LCWS 2009), Chicago, 2009.

[44] P. Schacht, ATLAS Liquid Argon Endcap Calorimeter R\&D for SLHC, ATL-LARG-PROC-2009-013, 2009.

[45] Z. Dolezal, Super KEKB and Belle II, Europhysics Conference on HEP, Krakow, 2009.

[46] M. Yokoyama et al., Application of Hamamatsu MPPCs to T2K neutrino detectors, Nucl. Instrum. Meth. A610 (2009) 128-130.

[47] Y. Kawai et al., Large-aperture hybrid photo-detector, Nucl. Instrum. Meth. A579 (2007) 42-45.

[48] Y. Kawai et al., Development of Large-Aperture Hybrid Avalanche Photo-Detector, 2009 IEEE Nuclear Science Symposium Conference Record (2009) 40-3.

[49] A. Amerio et al., Design, construction and tests of the ICARUS T600 detector, Nucl. Instrum. Meth. A527 (2004) 329-410.

[50] M. Pedretti et al., CUORE experiment: The search for neutrinoless double beta decay, Int. J. Mod. Phys. A23 (2008) 3395-3398.

[51] M.J. Dolinski, The Enriched Xenon Observatory, AIP Conf. Proc. 1182 (2009) 92-95.

[52] C.E. Aalseth et al., The Majorana Project, J. Phys. Conf. Ser. 203 (2010) 012057 [arXiv:0910.4598].

[53] C. Arnaboldi et al., Results from a search for the $0 v \beta \beta$-decay of ${ }^{130}$ Te, Phys. Rev. C78 (2008) 035502 [arXiv: 0802.3439 ].

[54] D.S. Akerib et al., Exclusion limits on the WIMP-nucleon cross section from the first run of the Cryogenic Dark Matter Search in the Soudan Underground Laboratory, Phys. Rev. D72 (2005) 052009. 
[55] T. Saab, Search for Weakly Interacting Massive Particles with the Cryogenic Dark Matter Search Experiment, Ph.D. Thesis, Stanford University (2002).

[56] Z. Ahmed et al., Results from the Final Exposure of the CDMS II Experiment, [arXiv:0912.3592].

[57] W.J. Bolte et al., Development of Bubble Chambers With Enhanced Stability and Sensitivity to Low-Energy Nuclear Recoils, Nucl. Instr. Meth. A 577 (2007) 569 [

arXiv:astro-ph/0503398v1].

[58] G. Sciolla and C.J. Martoff, Gaseous Dark Matter Detectors, New J.Phys.11:105018,2009 [arXiv:0905.3675].

[59] D. Dujmic et al., Charge amplification concepts for direction-sensitive dark matter detectors, Astropart. Phys. 30, 58 (2008).

[60] E. Aprile and T. Doke, Liquid Xenon Detectors for Particle Physics and Astrophysics, [arXiv:0910.4956].

[61] A. Hime, DEAP CLEAN detectors for low energy particle astrophysics, AIP Conf. Proc. 870 (2006) 205-207.

[62] K. Abe, The XMASS experiment, AIP Conf. Proc. 1182 (2009), 268-271.

[63] E. Aprile, The XENON dark matter project: Status of the XENON100 phase, AIP Conf. Proc. 1166 (2009) 205-211.

[64] S. Fiorucci et al., Status of the LUX Dark Matter Search [arXiv: 0912 . 0482].

[65] V. Boccone, Recent updates on the ArDM project: A Liquid Argon TPC for Dark Matter Detection, J. Phys. Conf. Ser. 160 (2009) 012032 [arXiv: 0810 . 4490]. 